\title{
Individual Differences in the Modulation of Fear-Related Brain Activation by Attentional Control
}

\author{
Andrew Mathews, Jenny Yiend, and Andrew D. Lawrence
}

\begin{abstract}
In this article, we consider the extent to which variations in the neural activation associated with fear-related stimuli are obligatory or optional. More specifically, we investigated modulation of activation according to type of encoding operation, and how this relates to individual differences in fearfulness and attentional control. In an fMRI study, fear-related (relative to neutral) pictures preferentially activated many of the regions involved in a hierarchical system responsible for organizing defensive behavior, and differential activation in some of these areas was related to self-reported individual variations in fearfulness. Preferential activation according to type of stimulus persisted to a limited extent even when attention was diverted from its emotional aspects. Importantly,
\end{abstract}

\section{INTRODUCTION}

Gray and McNaughton (2000; see also Fanselow, 1994) have proposed that defensive responses to threats are organized within a hierarchical system that includes the anterior cingulate, amygdala, hippocampus, and periaqueductal gray (PAG). Lower levels within this system are thought to control responses to imminent dangers, and anticipatory responses may involve higher levels (Barbas, Saha, Rempel-Clower, \& Ghashghaei, 2003; Keay \& Bandler, 2001). Thus, we assume that the term "fear" (and anxiety) can refer to the action of different parts of the whole system in response to danger, interacting in ways that may vary depending on specific circumstances, rather than always reflecting the action of only one specific component within it. Similarly, we will use the term "fear-related" to describe stimuli depicting dangers, which if encountered in reality would be expected to elicit fear, without assuming that they will all elicit activation in any one specific part of the system. We consider first the existing evidence relating to whether differential neural activation to fear-related versus neutral pictures is obligatory, or is influenced by optional attentional control processes. Much recent research has focused on the amygdala, although as indicated above,

MRC Cognition and Brain Sciences Unit, Cambridge, UK however, encoding tasks involving attention to emotional versus nonemotional attributes of the same pictures revealed a pattern of greater activation during emotional encoding, similar to that differentiating fear-related from neutral stimuli. Again, the degree of modulation varied according to individual differences. We conclude that fear-related pictures can recruit activation in the defensive system even when attention is directed elsewhere, but that the extent of this activation is modulated by attentional control mechanisms. More critically, both differential activation and its modulation by attentional control are related to individual variations in emotional vulnerability, in a manner that conforms to predictions derived from existing theoretical accounts.

we suppose that other parts of the fear system might also respond in an obligatory or optional fashion. Then, we consider if such obligatory or optional processes are related to individual variations in reported fearfulness and attentional control.

Fearful (relative to neutral) faces elicit more activation in areas within the fear system (such as the amygdala), even when they are not the focus of attention (Anderson, Christoff, Panitz, De Rosa, \& Gabrieli, 2003; Iidaka et al., 2001; Vuilleumier, Armony, Driver, \& Dolan, 2001). This effect persists when faces are backward masked by superimposition of neutral stimuli (Öhman, 2002; Morris, Öhman, \& Dolan, 1999; Whalen et al., 1998), and following lesions to the visual cortex resulting in functional blindness ("affective blindsight": Hamm et al., 2003; Morris, DeGelder, Weiskrantz, \& Dolan, 2001; although see also Heywood \& Kentridge, 2000). Such data can be seen as consistent with a proposed subcortical visual fear pathway, bypassing the need for higher-level cognitive processing (Shi \& Davis, 2001; Doron \& Ledoux, 1999).

Although such studies have been interpreted (e.g., by Dolan \& Vuilleumier, 2003; Öhman \& Mineka, 2001) as showing that fear-related activation is obligatory, other evidence suggests that fear-related processing can be influenced by attentional control operations. Several recent studies have shown that activation in brain areas implicated in fear-related defensive responses can be 
modified by instructions to enhance or to suppress emotional reactions to aversive pictures (Ochsner, Bunge, Gross, \& Gabrieli, 2002; Schaefer et al., 2002; Jackson, Malmstadt, Larson, \& Davidson, 2000). However, interpretation of these studies is complicated by a lack of experimental control over what participants actually did to modify their emotion. For example, it is difficult to be certain about what aspects of picture content were actually attended and encoded.

Other neuroimaging studies have achieved better experimental control over attention and encoding processes by requiring participants to make a specific judgement about the content of the evoking stimuli. Simply making an emotional or nonemotional judgement about fear-related pictures can reduce the activation in fear-related areas such as the amygdala seen during passive viewing (Lange et al., 2003; Taylor, Phan, Decker, \& Liberzon, 2003). Matching to verbal labels, versus other pictures, also decreases amygdala activation (Hariri, Mattay, Tessitore, Fera, \& Weinberger, 2003; Hariri, Bookheimer, \& Mazziotta, 2000), whereas making emotional ratings enhances activation, relative to recognition (Liberzon et al., 2000), or age judgements (Iidaka et al., 2001). In a study contrasting emotional with nonemotional ("inside-outside") judgements of scenes, Lane, Fink, Chau, and Dolan (1997; see also Gusnard, Akbudak, Shulman, \& Raichle, 2001) found more anterior cingulate, medial prefrontal, and hippocampal (but not amygdala) activation in the former. Keightley et al. (2003) reported a more complex pattern of activation that was greater when making emotional judgements of scenes versus counting the number of people present, but (confusingly) the same activation pattern was "less" marked when making emotional versus gender judgements about faces. The strongest claim, to the effect that attention-demanding judgements can actually eliminate fear-related activation, is due to Pessoa, McKenna, Gutierrez, and Ungerleider (2002). The usual differential amygdala response to fearful versus neutral faces seen during gender judgements was apparently eliminated when participants had to make difficult perceptual judgements about unrelated flanking stimuli.

By and large, these studies have not involved any investigation of a possible role for individual differences, although there is a great deal of evidence suggesting that mood states can indeed have important effects (e.g., Zald, 2003). However, rather than mood states per se, we are concerned here with more persistent traitlike differences that predispose people to react differently to the same fear-related stimuli. In fact, there have been surprisingly few neuroimaging studies of emotional trait differences, and virtually none in which these differences have been related to activation by fear-related stimuli.

The neuroimaging studies most directly relevant to this issue are those of Canli, Sivers, Whitfield, Gotlib, and Gabrieli (2002) and Canli, Zhao, et al. (2001), in which participants were exposed to pleasant and unpleasant pictures. Variations in extraversion were correlated with increased activation due to pleasant versus unpleasant pictures in several brain areas, including some in the frontal and temporal cortices, the amygdala, and the putamen. By contrast, neuroticism (general vulnerability to unpleasant emotional states) was related to greater activation by unpleasant versus pleasant pictures in the left middle temporal and frontal gyri (see also Gusnard, Ollinger, et al., 2003; Schwartz, Wright, Shin, Kagan, \& Rauch, 2003).

In the present study, we were concerned with the more specific traits of anxiety and proneness to fear. According to Gray and McNaughton (2000), such individual variations depend on the action of a septohippocampal behavioral inhibition system, that engages the amygdala to produce fear-related outputs. The Behavioral Inhibition Scale (BIS, Carver \& White, 1994) is a self-report measure specifically designed to assess these individual differences (see Methods for details). We therefore investigated whether BIS scores are related to the extent of hippocampal and amygdala activation when viewing fear-related stimuli. Anxietyprone individuals are known to be persistently attentive to fear-related stimuli (Yiend \& Mathews, 2001; Mathews \& MacLeod, 1994). This effect has been modeled as the outcome of two mutually inhibitory processes: threat-related signals arising within lower regions of the defensive system, and top-down attentional control (Derryberry \& Reed, 2002; Drevets \& Raichle, 1998; Mathews, Mackintosh, \& Fulcher, 1997). For this reason, we also assessed individual variations in self-reported ability to control attention, and inhibit unwanted distractions, using the Attentional Control Questionnaire (ACQ, Derryberry \& Reed, 2002). Regions within the defensive system considered to be important in the attentional control of responses to threat ("coping") include the rostral anterior cingulate and medial prefrontal cortices (Barbas et al., 2003; Keay \& Bandler, 2001; Bush, Luu, \& Posner, 2000; Gray \& McNaughton, 2000).

We required participants to judge whether pictures were more frightening than the previous one (emotional encoding), or had required more planning or preparation on the part of the photographer (nonemotional encoding). Some of the pictures had been selected to be fear-related (i.e., depicting a potentially dangerous situation) whereas the remainder were emotionally neutral, but with similar perceptual content and complexity.

No judgments were required in an initial baseline uninstructed viewing condition. Areas differentially activated by fear-related and neutral pictures in this baseline condition were then used to define regions of interest (ROIs) for the purposes of correlations with individual differences, and for comparisons across other conditions in which encoding instructions were specified. Across the instructed encoding conditions, presented in fully 
counterbalanced order, one contrast was between fearrelated and neutral pictures, holding the same (nonemotional) encoding task constant, with the intention of revealing any obligatory emotional activation to fearrelated pictures. The other contrast was between emotional and nonemotional encoding conditions, holding the same (fear-related) picture content constant, with the intention of revealing any optional effects due to top-down control.

\section{RESULTS}

\section{Behavioral Data}

The mean latencies to make emotional and nonemotional judgement decisions for the fear-related pictures were computed for each participant. There were no significant differences between the mean latencies for each type of encoding decision, $t(22)=0.66, p=.52$ (emotional judgement mean 2018 msec, nonemotional $2045 \mathrm{msec}$ ), consistent with our intent that the tasks should make similar attentional demands. We also computed the reliability between the "yes-no" encoding judgements made across repeated presentations in each type of task, for each participant, using Cronbach's alpha. The mean coefficient was 0.74 (SD 0.16), demonstrating that participants were reasonably consistent in repeating the same decision across two occasions.

The two questionnaires used (the BIS and the ACQ) were not significantly correlated with each other across the whole sample, $r(22)=.10, p=.6$. Analyses of variance of the latencies to carry out each of the encoding judgements, with above or below median scores on each of the above questionnaires as grouping factors, did not reveal any significant effects. Finally, we contacted the participants 6 months later (only 15 could be traced) and asked them to recall any pictures they remembered seeing. The proportion of correct hits (scored by a single blind assessor) was greater for fearrelated than neutral pictures $(4.7 \%$ vs. $1 \%, p<.001)$ but correlations between this difference and questionnaire scores did not approach significance.

\section{Functional MRI Data}

Comparisons across Picture Types during Uninstructed Viewing

Analyses comparing activation during uninstructed viewing of fear-related versus neutral pictures revealed a number of brain regions where activation was significantly greater for fear-related than for neutral pictures. These included areas within the amygdala, hippocampus, parahippocampal cortex, visual cortex, dorsomedial prefrontal cortex, rostral anterior cingulate cortex, pre/ postcentral gyrus, insula, posterior cingulate, perirhinal cortex, pulvinar nucleus of the thalamus, superior colliculus, and PAG (see Figure 1 and Table 1a). No areas were found to be significantly more activated by neutral than by fear-related pictures.

To explore the hypothesis that greater activation to fear-related pictures should occur in those with high BIS scores, we regressed activity for the fear minus neutral picture contrast against BIS scores. This analysis revealed that the enhanced activation by fear-related versus neutral pictures was more pronounced in those with high BIS scores in several of the above locations: specifically, the hippocampus/parahippocampal cortex, amygdala, pulvinar, and PAG (see Figure 2 and Table 1b). Thus, as expected, those individuals reporting greater vulnerability to threat did indeed respond with relatively greater activation in those areas sensitive to fear-related versus neutral pictures. Also, scores on the ACQ were correlated with differential activation due to fear-related versus neutral pictures in the rostral anterior cingulate cortex.

\section{Comparison across Picture Types with Nonemotional Encoding}

Results of analysis comparing activation due to picture type, holding the (nonemotional) encoding task constant, showed more activation to fear-related than neutral pictures in the visual cortex, similar to that seen during uninstructed viewing. Also as before, fear-related pictures were associated with greater activation of the
Figure 1. (From left to right) BOLD response in (A) the left amygdala (MNI $x, y, z=-22$, $-10,-18)$; (B) the left hippocampus (MNI $x, y$, $z=-28,-26,-18)$; (C) the midbrain PAG region (MNI $x, y$, $z=-4,-36,-26)$, for the contrast (freeview fear-related minus neutral images). Activations thresholded at $p_{\text {FDR }}<.05$, minimum cluster extent 5 voxels, and displayed on coronal slices of the "avg152T1_brain" structural template image of SPM 99.

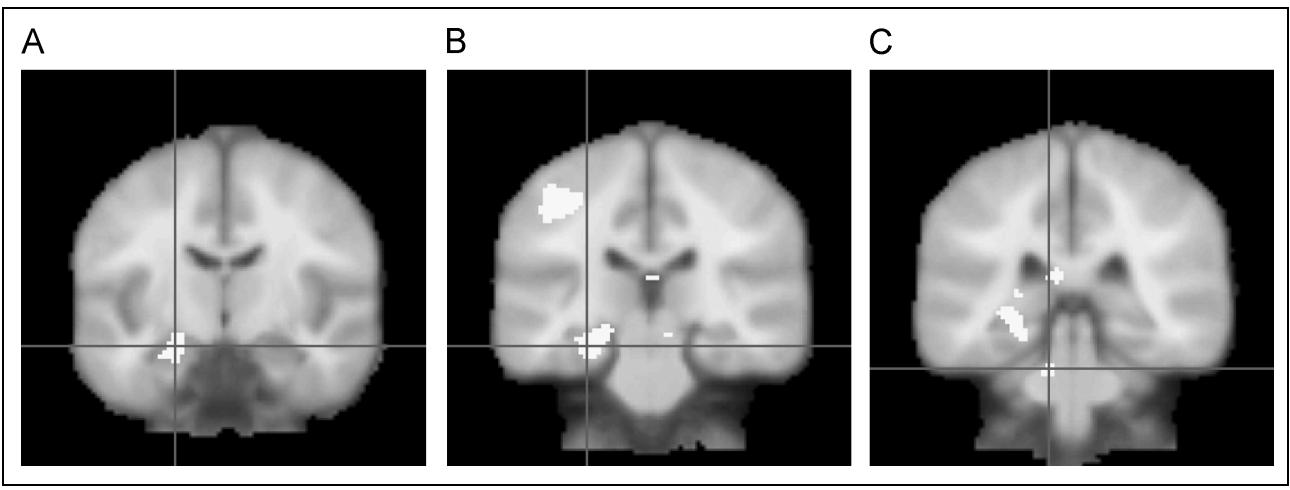


Table 1. Regions of Activation during Passive Viewing of Fear-Related Pictures

(a) Brain regions (with MNI coordinates) showing greater activation during passive viewing of fear-related relative to neutral pictures. Here we report activation foci that survive a whole brain false discovery rate (FDR) correction at $p<.05$, with a minimum cluster extent of 5 voxels. Note that, since the FDR correction is an adaptive procedure, statistical thresholds are at different values for the different contrasts. $L=$ left; $R=$ right

\begin{tabular}{|c|c|c|c|c|}
\hline & \multicolumn{3}{|c|}{ Coordinates } & \multirow[b]{2}{*}{$Z$ score } \\
\hline & $x$ & $y$ & $z$ & \\
\hline R Lingual gyrus & 16 & -88 & -8 & 5.60 \\
\hline L Lingual gyrus & -10 & -98 & -10 & 5.39 \\
\hline R Cuneus & 12 & -96 & -12 & 4.29 \\
\hline R Occipital gyrus & 34 & -88 & -12 & 3.17 \\
\hline L Inferior temporal gyrus & -44 & -70 & -70 & 3.17 \\
\hline R Amygdala & 20 & -2 & -24 & 3.21 \\
\hline L Amygdala & -22 & -10 & -18 & 3.93 \\
\hline L Pre/postcentral gyrus & -42 & -22 & 52 & 4.38 \\
\hline L Pre/postcentral gyrus & -46 & -22 & 44 & 4.15 \\
\hline $\begin{array}{l}\text { L Hippocampus/ } \\
\text { perirhinal cortex }\end{array}$ & -20 & -30 & -12 & 3.90 \\
\hline L Hippocampus & -28 & -26 & -18 & 3.80 \\
\hline $\begin{array}{l}\text { R Hippocampus/ } \\
\text { parahippocampal cortex }\end{array}$ & 40 & -16 & -24 & 3.36 \\
\hline L Thalamus (lp/pulvinar) & -6 & -36 & 14 & 3.87 \\
\hline R Thalamus (lp/pulvinar) & 2 & -26 & 14 & 3.19 \\
\hline L Pulvinar & -22 & -34 & 6 & 3.16 \\
\hline R Superior colliculus & 8 & -28 & -12 & 3.20 \\
\hline L Insula & -34 & -2 & 14 & 3.71 \\
\hline L Periaqueductal gray & -10 & -34 & -28 & 3.63 \\
\hline L Rostral anterior cingulate & -12 & 50 & 2 & 3.56 \\
\hline L Rostral anterior cingulate & -2 & 64 & 8 & 3.26 \\
\hline L Posterior cingulate & -2 & -22 & 34 & 3.18 \\
\hline L Dorsomedial prefrontal & -12 & 46 & 24 & 3.24 \\
\hline
\end{tabular}

(b) Regions in which differences were positively correlated with BIS scores. $p<.05$, FDR, small volume correction based on coordinates from the above contrast, minimum cluster extent 5 contiguous voxels.

\begin{tabular}{lrrrr}
\hline $\begin{array}{l}\text { R Hippocampus/ } \\
\text { parahippocampal cortex }\end{array}$ & 40 & -16 & -12 & 2.44 \\
R Hippocampus & 16 & -34 & -8 & 2.23 \\
R Amygdala & 14 & 2 & -16 & 2.42 \\
R Amygdala & 14 & -6 & -26 & 2.09
\end{tabular}

Table 1. (continued)

\begin{tabular}{lrrrr}
\hline & \multicolumn{3}{c}{ Coordinates } \\
\cline { 2 - 4 } & $x$ & $y$ & $z$ & $Z$ \\
\hline R Pulvinar & 4 & -34 & 12 & 3.96 \\
L PAG & -4 & -36 & -26 & 3.21 \\
(As above, for ACQ scores) & & & & \\
Rostral ACC & -16 & 52 & -4 & 2.91 \\
\hline
\end{tabular}

amygdala, although this was now significant only on the left side and at $p<.07$ (see Figure 3 and Table 2a). No other brain region revealed significant differences.

Only one of the areas previously found to distinguish between fear-related and neutral pictures remained significantly correlated with individual differences in BIS scores: a region in the right hippocampus/hippocampal cortex (Table 2b). There were no significant correlations with attentional control scores. Exploratory analyses of other areas outside the designated ROIs, using whole brain correction, revealed no additional significant results in these or subsequent analyses.

\section{Comparison across Different Types of Encoding for Fear-Related Pictures}

Results of an analysis contrasting emotional with nonemotional encoding of fear-related pictures revealed differential activation in brain regions that overlapped considerably with those previously found to distinguish between fear-related and neutral pictures. Specifically, emotional encoding was associated with greater activa-

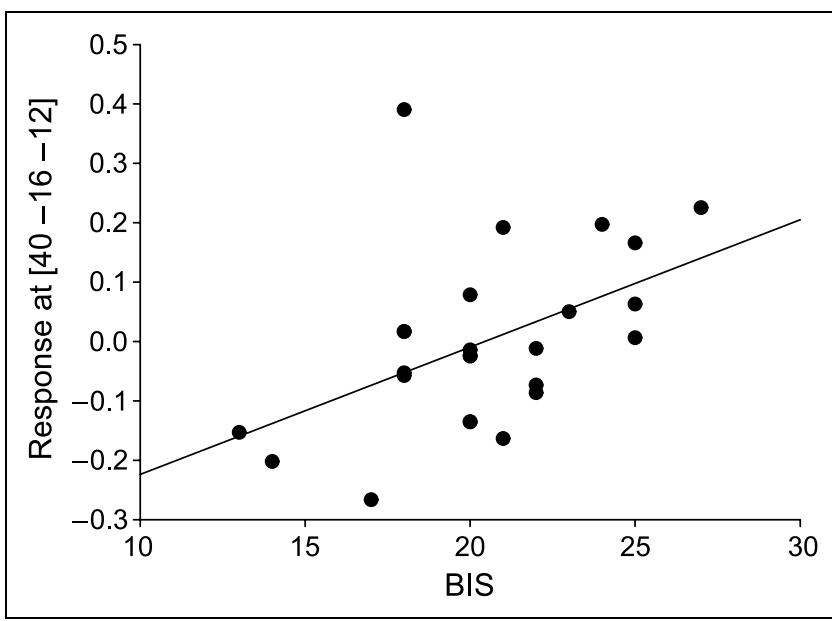

Figure 2. Scatterplot showing BOLD response in the right hippocampus/parahippocampal cortex (MNI $x, y, z=40,-16,-12$ ) as a function of BIS score for the contrast (freeview fear-related minus neutral images). 


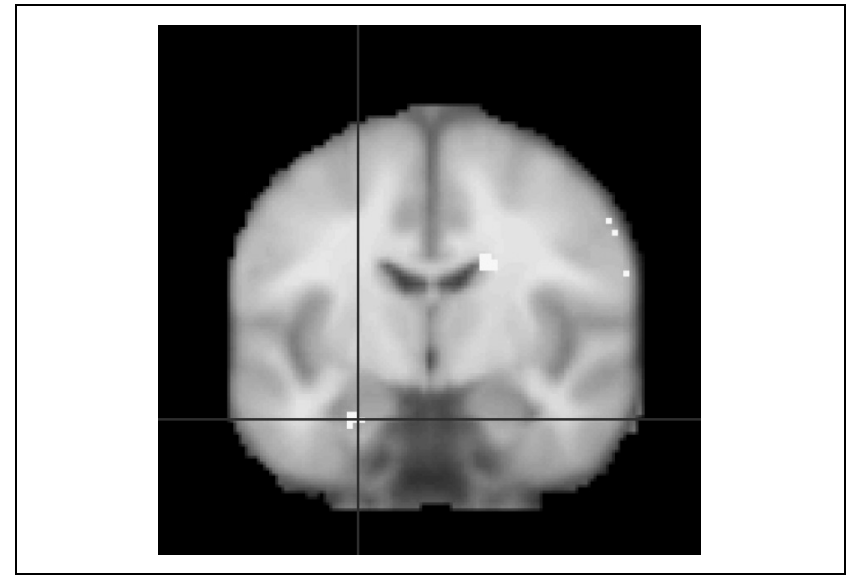

Figure 3. BOLD response in the left amygdala (MNI $x, y, z=-24$, $-8,-26)$ for the contrast (nonemotional encoding of fear-related vs. neutral images). Activations thresholded at $p_{\text {uncorrected }}<.1$, minimum cluster extent 5 voxels, and displayed on a coronal slice of the "avg152T_brain" structural template image of SPM 99.

tion in the visual cortex (Figure 4), the dorsomedial prefrontal and rostral anterior cingulate cortex (Figure 5), the amygdala, the hippocampus, and the pulvinar. Thus, optional cognitive encoding processes significantly modulated the extent of activation in many of the areas previously found to be differentially sensitive to fearrelated versus neutral pictures during uninstructed viewing (see Table 3).

Table 2. Regions of Activation during Nonemotional Encoding of Fear-Related Pictures

(a) Brain regions showing greater activation during nonemotional encoding of fear-related versus neutral pictures. Activations thresholded at $p<.05$, FDR, small volume (12 $\mathrm{mm}$ radius sphere) corrections based on coordinates shown in Table 1a, minimum cluster extent of 5 contiguous voxels.

\begin{tabular}{lcccc}
\hline & \multicolumn{3}{c}{ Coordinates } & \\
\cline { 2 - 4 } Region & $x$ & $y$ & $z$ & \multirow{2}{*}{ Z score } \\
\hline L Lingual gyrus & -16 & -92 & -6 & 4.08 \\
L Occipital gyrus & -24 & -90 & -8 & 2.52 \\
L Fusiform gyrus & -38 & -68 & -16 & 2.08 \\
L Amygdala & -24 & -8 & -26 & $1.50^{*}$
\end{tabular}

(b) Regions in which differences were positively correlated with BIS scores. $p<.05$, FDR, SVC based on coordinates from Table 1a, minimum cluster extent 5 voxels.

\begin{tabular}{lllll}
\hline $\begin{array}{l}\text { R Hippocampus/ } \\
\text { parahippocampal cortex }\end{array}$ & 46 & -6 & -24 & 2.14 \\
\hline
\end{tabular}

$* p<.07$.

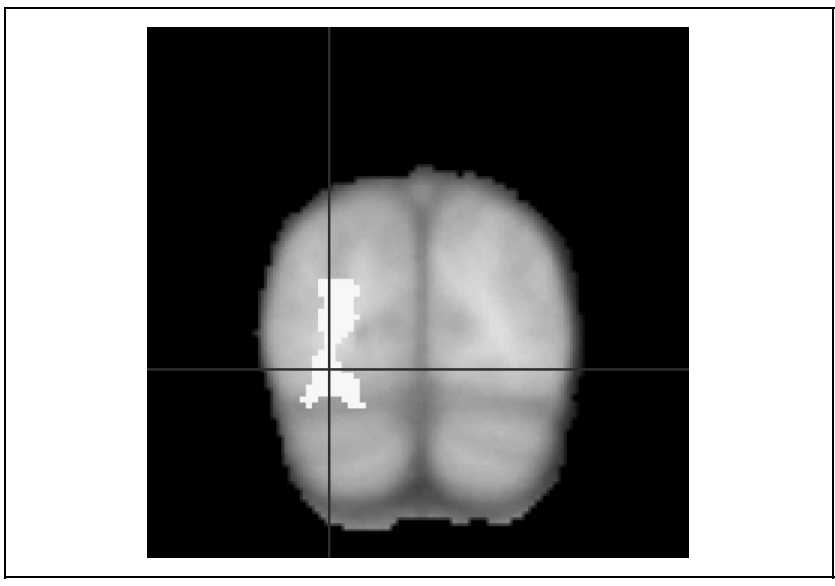

Figure 4. BOLD response in the left occipital lobe (MNI $x, y$, $z=-30,-80,-8)$ for the contrast (emotional vs. neutral encoding of fear-related images). Activations thresholded at $p_{\text {uncorrected }}<.05$, minimum cluster extent 5 voxels, and displayed a coronal slice on the "avg152T_brain" structural template image of SPM 99.

Activation differences due to the type of encoding, in the previously defined regions of interest, were again tested for correlations with BIS scores across individuals. Two significant positive correlations remained; one with the region in the hippocampus/parahippocampal cortex, and the other with the PAG. That is, the greater activation of these two areas due to emotional versus nonemotional encoding was more pronounced in those individuals reporting higher responsiveness to threat on the BIS. Additionally, however, BIS scores were significantly correlated with rostral anterior cingulate cortex activation in a negative direction (see Figure 6). In contrast, ACQ scores correlated positively with activation in the same area. Thus, differential activation of the rostral anterior cingulate was greater in those with lower BIS scores, and in those reporting high attentional control.

\section{DISCUSSION}

First, in the baseline condition, we confirmed that fearrelated pictures activated areas in the visual cortex more than did neutral pictures (Bradley et al., 2003; Lang, Bradley, Fitzsimmons, et al., 1998). More critically, fearrelated pictures activated essentially the whole of the hierarchical defensive system described by Gray and McNaughton (2000) and Fanselow (1994). Activation involved both cortical (thalamo-cortico-amygdaloid) and subcortical (thalamo-amygdaloid) routes, whereby visual fear-related stimuli can provide input to the amygdala (Shi \& Davis, 2001), as well as cortical and subcortical networks important for defensive reactions (Cooke \& Graziano, 2004; Bradley \& Lang, 2000; Shi \& Cassell, 1998; McNish, Gewirtz, \& Davis, 1997).

Furthermore, differential activation in some of these areas (hippocampus/parahippocampal cortex, amygdala, pulvinar, and PAG) was associated with BIS scores. This 
Figure 5. BOLD response in the rostral anterior cingulate cortex (MNI $x, y, z=-6,44,6)$ and the medial prefrontal cortex for the contrast (emotional vs. nonemotional encoding of fear-related images). Activations thresholded at $p_{\text {uncorrected }}$ $<.05$, minimum cluster extent 5 voxels, and displayed on a sagittal (left image) and coronal (right image) slice of the "avg152T1_brain" template image of SPM 99

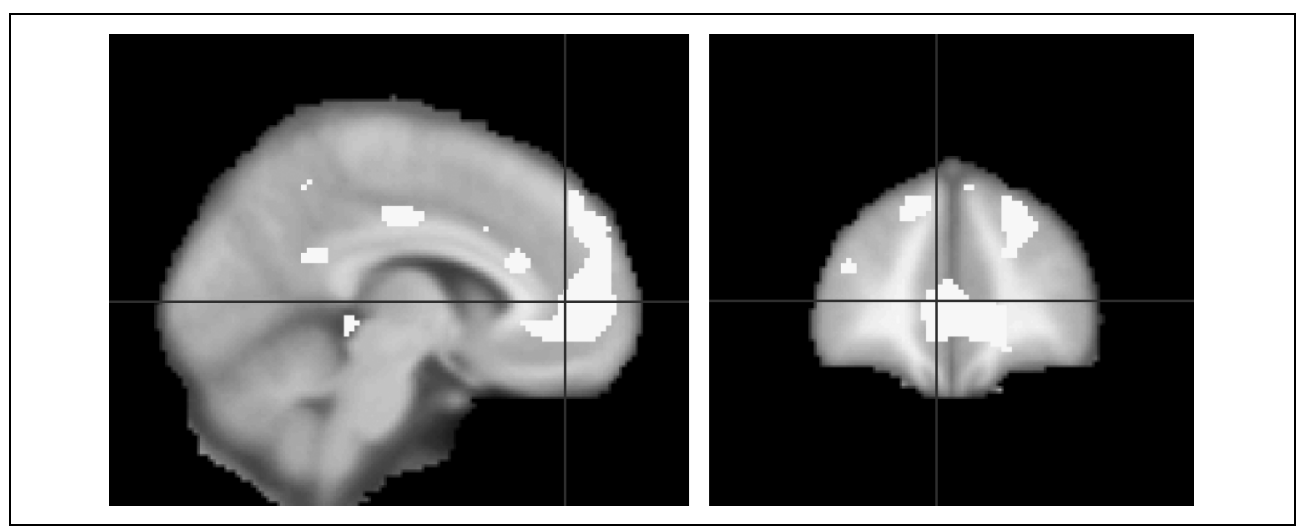

provides strong evidence for the argument of Gray and McNaughton (2000), that variable input from hippocampus to other parts of the defensive system underlies trait differences in vulnerability to anxiety. Lesions to the hippocampus in animals impair a number of fear and anxiety related behaviors (Bannerman et al., 2003; Sanders, Wiltgen, \& Fanselow, 2003; Deacon, Bannerman, \& Rawlins, 2002; Kjelstrup et al., 2002). In particular, hippocampal and PAG activation are involved in freezing behavior in animals (McNish et al., 1997) and it has been suggested that the slowed disengagement of attention from threat cues in humans could arise similarly (Fox, Russo, Bowles, \& Dutton, 2001).

There was also evidence of differential activation within rostral anterior cingulate and dorsomedial frontal areas, supposedly at the top of the hierarchical defense system, and involved in the flexible regulation of defensive behaviors (Barbas et al., 2003; Keay \& Bandler, 2001; Gray \& McNaughton, 2000). The extent of differential activation in the rostral anterior cingulate/dorsomedial prefrontal cortex due to fear-related pictures was positively correlated with ACQ scores. Activation of this area has been noted when emotional words are to be ignored (Bush et al., 2000); and when participants attend to their emotional reactions (Gusnard, Akbudak, et al., 2001; Lane, Fink, et al., 1997). Despite not being required to do so, it is possible that participants with higher control scores attended more to their own emotional experience, or were more active in controlling their attention to fear-related pictures. This latter suggestion is consistent with findings that lesions to the dorsomedial PFC can enhance fear (Morgan \& LeDoux, 1995; see also, Quirk, Likhtik, Pelletier, \& Pare, 2003).

The comparison between fear-related and neutral pictures, holding nonemotional encoding constant, was designed to assess whether differential activation would persist when attention was directed to nonaffective attributes. Differential activation persisted in the visual cortex and (albeit weakly) in the left amygdala. These findings support suggestions that attention to nonemotional attributes does not abolish the greater amygdala response to fear-related stimuli (e.g., Anderson et al., 2003). The abolition of differential amygdala activation reported by Pessoa et al. (2002) occurred in the context of a very perceptually demanding task. Under high perceptual load, processing of spatially separated distracters can be compromised (Lavie, 1995) and emotional stimuli are apparently no exception to this rule. When fear-related stimuli are in the spatially attended location, however, we take the present results to indicate that some enhanced sensory and amygdala response to fearrelated pictures persists, despite attention to nonaffective attributes.

Some parts of the defensive system (such as the amygdala) may thus respond to fear-relevant stimuli in a relatively obligatory fashion, whereas the reduced differential response in most other areas (such as frontal cortex) suggests more optional responding. Optional cognitive encoding processes exerted a modulating influence even over those areas thought to form part of a subcortical visual pathway to the amygdala (i.e., pulvinar and superior colliculus; see Quirk et al., 2003; Keay \& Bandler, 2001). Similarly, most of the correlations with BIS scores seen during uninstructed viewing were no longer significant during nonemotional encoding, leaving only that between BIS and hippocampal activation. Thus, both differential activation to fearrelated pictures and its enhancement in high BIS individuals can be modulated by cognitive control mechanisms. If the enhanced reactivity of individuals with high BIS scores can be partly overridden by instructions, it cannot be fully obligatory. The one exception may indicate that differential activation of the hippocampus is more resistant to control. Within the GrayMcNaughton theory, this suggests that the hippocampus is more likely to continue signaling the presence of potential threat in vulnerable individuals, regardless of the current task.

In the final comparison, we were specifically concerned with the extent to which optional encoding processes modulate the brain activation associated with fear-related pictures. Strikingly, some neural areas more 
Table 3. Regions of Activation during Emotional Encoding of Fear-Related Pictures

(a) Brain regions showing greater activation to fear-related pictures during emotional versus nonemotional encoding (criteria as for Table 2.)

\begin{tabular}{lrrrr}
\hline & \multicolumn{3}{c}{ Coordinates } & \\
\cline { 2 - 4 } Region & $x$ & $y$ & $z$ & $Z$ \\
\hline L Lingual gyrus & -24 & -82 & -14 & 3.27 \\
L Lingual gyrus & -20 & -96 & -8 & 2.26 \\
L Occipital gyrus & -30 & -80 & -8 & 3.94 \\
L Inferior temporal gyrus & -46 & -72 & -16 & 2.95 \\
L Amygdala & -20 & -14 & -12 & 1.79 \\
R Amygdala & 18 & 8 & -24 & 2.16 \\
L Hippocampus & -22 & -22 & -12 & 1.95 \\
L Hippocampus & -38 & -12 & -16 & 3.41 \\
R Pulvinar & 4 & -32 & -2 & 2.01 \\
L Insula & -34 & 4 & 6 & 2.18 \\
L Rostral ACC & -6 & 44 & 6 & 3.96 \\
L Rostral ACC & -4 & 56 & 0 & 3.71 \\
L Posterior cingulate & -2 & -18 & 40 & 3.58 \\
L Dorsomedial PFC & -8 & 54 & 32 & 3.00
\end{tabular}

(b) Regions in which differences were positively correlated with BIS scores (criteria as for Table 2)

\begin{tabular}{lrrrr}
\hline R Hippocampus/ & 40 & -16 & -22 & 2.46 \\
$\quad$ Parahippocampal cortex & & & & \\
L PAG & -2 & -40 & -24 & 2.31 \\
L Rostral ACC & -8 & 46 & 2 & $3.02 *$ \\
(As above, for ACQ scores) & & & & \\
R Rostral ACC & 2 & 40 & 2 & 3.88 \\
L Rostral ACC & -16 & 52 & 4 & 1.76 \\
\hline
\end{tabular}

*Correlation of ACC with BIS is negative.

sensitive to fear-related than neutral pictures were also modulated by type of encoding. That is, optional encoding processes modulated activation within parts of the defensive system-specifically, the amygdala and the hippocampus (as well as the visual cortex and the pulvinar) - that were sensitive to the fear-neutral contrast. Differences in visual cortex activation have been attributed to back projections from the amygdala (Amaral, Behniea, \& Kelly, 2003; Bradley et al., 2003; Schupp, Junghöfer, Weike, \& Hamm, 2003; Lang, Bradley, Fitzsimmons, et al., 1998; Lane, Reiman, et al., 1997). The amygdala can also influence the visual cortex via the ba- sal forebrain cholinergic system (Holland, Han, \& Gallagher, 2000; Kapp, Whalen, Supple, \& Pascoe, 1992). Heightened visual cortical activation to fear-related stimuli would place them at a competitive advantage for attention. Whereas previous findings could be attributed to perceptual rather than emotional attributes of different picture sets; in the present study, differential activation occurred due to type of encoding deployed with the same picture sets, ruling out explanation in terms of differing perceptual characteristics.

The finding that hippocampal and amygdala activation was also significantly modulated by nonemotional versus emotional encoding instructions is consistent with previously reported effects of attention to emotional or nonemotional attributes of the same (emotional) stimuli (e.g., Gur et al., 2002). Problems of interpretation can arise, however, if the number of pictures presented, or the processing demands of the encoding task, is not well controlled. Thus, increasing the demand placed on working memory reduces the response to a fear-related conditioned stimulus (Carter, Hofstotter, Tsuchiya, \& Koch, 2003). In the present case, we ensured that participants attended to the same pictures across encoding conditions, and required judgements of the whole picture that took similar processing time, implying similar difficulty levels. Finally, by using one-back comparisons, we ensured that working memory load was relatively constant. With these aspects controlled, our results indicated that encoding aspects of meaning related to fear leads to greater amygdala and hippocampal activation than does encoding nonemotional aspects of the same stimuli.

As before, BIS scores were correlated with differential hippocampal activation, but now due to the type of encoding for the same pictures. Individuals with high BIS scores showed relatively more activation in both the

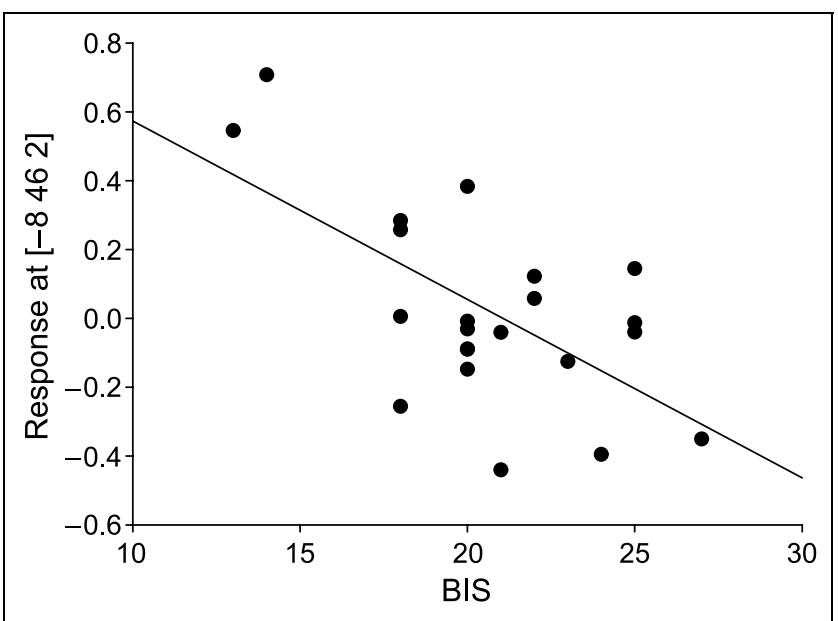

Figure 6. Scatterplot showing BOLD response in the rostral anterior cingulate cortex (MNI $x, y, z=-8,46,2$ ) as a function of BIS score for the contrast (emotional vs. nonemotional encoding of fear-related images). 
hippocampus and the PAG when making emotional versus nonemotional judgements. BIS scores were negatively correlated with differential activation in the rostral anterior cingulate, however, perhaps indicating less attentional control over threat signals (Bush et al., 2000). The positive association between differential activation in the same area and ACQ scores is consistent with this explanation. Both high control and low vulnerability to threat are thus associated with greater activity in medial frontal regions, presumably because both are associated with more effective top-down modulation of the lower regions of the defensive behavior system, including the PAG (Quirk et al., 2003; Keay \& Bandler, 2001; Morgan \& LeDoux, 1995).

In summary, our evidence reveals persistence of fearrelated differential activation in the amygdala and the visual cortex, even when attending to nonemotional aspects of pictorial stimuli. This may be thought consistent with the view that activity in the neural system underlying fear is relatively obligatory, and resists cognitive control (e.g., Öhman \& Mineka, 2001). In contrast, however, there was very clear evidence that fear-related activation was modulated by optional encoding processes, indicating that it is not immune from the influence of attentional control (cf. Pessoa et al., 2002). Areas modulated by attentional control include those within a proposed subcortical route, sometimes thought of as being more automatic. Importantly, both the magnitude of the fear-related activation seen, and the degree to which it was modulated by optional encoding processes, varied according to threat vulnerability (BIS) in a manner consistent with predictions from the theory proposed by Gray and McNaughton (2000). We suggest that fear-related stimuli generally have a competitive processing advantage over more neutral stimuli, particularly in those individuals reporting high levels of fearfulness, but not in a completely obligatory fashion as fear-related activation varies according to other cognitive processing demands.

\section{METHODS}

\section{Participants}

Of 27 volunteers recruited from the MRC-CBU volunteer panel, data from three were lost: one due to equipment failure and another two because they were unable to continue due to excessive anxiety. The number included in the final sample were thus 24 in the uninstructed viewing condition, but incomplete fMRI datasets for the encoding conditions reduced numbers to 22, of whom 16 were women. All had English as their first language, were right-handed, and had adequate vision to read instructions visible in a mirror mounted above their head when lying in the MRI scanner. None had a history of neurological illness, were currently taking psychotropic medication or receiving other treatment for a psychiatric illness. We attempted to recruit participants with above average as well as average or low levels of anxiety (on the basis of prior testing), so as to ensure a wide range of BIS scores. The study was approved by the Addenbrooke's Local Research Ethics Committee (Cambridge, UK) and written informed consent was obtained from all participants.

\section{Materials}

Pictures (114) were selected from the IAPS set (Lang, Bradley, \& Cuthbert, 1999), with 72 being clearly related to fear (high normative ratings on negative valence and arousal) and 42 being neutral or very mildly pleasant. The content of pictures in the fear-related and neutral sets was individually matched for nonemotional content. For example, a picture of a large snarling dog was matched with another of a docile-looking dog, and a picture of an assault in progress was matched with a picture of an affectionate couple. We also matched fear and neutral pictures for a presence and number of people present. In pilot work we asked six judges to decide if each picture was fear-related or otherwise as quickly as they could. Selected pictures were all categorized correctly and mean decision latencies were similar across the counterbalanced picture sets.

Twenty-four pictures were used in the baseline task, 12 fear-related and 12 neutral, each presented for $2 \mathrm{sec}$ followed by an interval of $3 \mathrm{sec}$. There were four blocks, each of six pictures of the same type, with block-order (threat-neutral or neutral-threat), and the assigned picture sets, being counterbalanced across participants. Each block of six pictures lasted $30 \mathrm{sec}$, before switching to a block with a different type of picture. The whole task was then repeated in the same order, so that each picture was seen twice.

Ninety pictures were used in the instructed encoding tasks, 60 fear-related and 30 neutral. Encoding instructions for each block were displayed for $5 \mathrm{sec}$, followed by five pictures of the same type, each displayed for $2 \mathrm{sec}$, followed by a 3-sec period in which a judgement prompt was displayed. Other than the first (reference) picture in each block, participants pressed a yes-no button to indicate whether the currently viewed picture represented a more frightening situation than the last one seen, or would have required more planning or preparation to obtain, depending on instructions given for that block. The 90 pictures were presented within 18 blocks, such that 12 of the blocks contained fearrelated pictures ( 6 with emotional and 6 with nonemotional encoding instructions), and 6 blocks contained neutral pictures (with nonemotional encoding instructions). After all the pictures had been viewed, there was a short pause before the same sequence was repeated, so that each picture was seen and rated twice.

The BIS (Carver \& White, 1994) is a seven-item questionnaire designed to reflect variations in fearful 
responding to threat (e.g., "If I think something unpleasant is going to happen I usually get pretty worked up," followed by a 4-point scale to rate agreement). Carver and White demonstrated that the BIS was reliable $(r=.66)$, correlated significantly $(r=.58)$ with the Manifest Anxiety Scale, and was a better predictor than the MAS of nervousness when anticipating a painful experience. In a large community sample, Jorm et al. (1998) used factor analysis to confirm that the BIS items emerged as a unitary factor, was reliable (Cronbach's alpha of .76), and correlated significantly with other measures of negative affect (e.g., with the Neuroticism scale of Eysenck Personality Questionnaire, $r=.64$ ). The complementary Behavioral Approach Scale (BAS) was also administered, but BAS scores did not predict BOLD signal changes for any contrast of interest at the $p<.05$ whole brain corrected (FDR) level. The ACQ (Derryberry \& Reed, 2002) was designed to tap variations in the perceived ability to direct attention (e.g., "When concentrating, I can focus my attention so that I become unaware of what's going on in the room around me"). Derryberry and Reed (2002) demonstrated that ACQ scores were significantly associated with an objective measure of the time taken to disengage attention from salient distracters.

\section{Procedure}

After a screening interview, the nature of the pictures and judgment to be used were explained to participants, and informed written consent obtained. A short practice task was then administered using a laptop computer, in which 35 pictures (not used subsequently) were presented to accustom participants to the task, and ensure that they understood the judgements required. Once positioned in the magnet, participants carried out the baseline task, lasting $4 \mathrm{~min}$, followed by two runs of the instructed encoding task, each lasting for 9 min. After structural images had been obtained, participants left the MRI room, and completed the BIS/BAS scales, and the ACQ.

\section{Scanning Procedure}

BOLD (blood oxygenation-level dependent) contrast functional images were acquired with echo-planar T2* weighted (EPI) imaging using a Medspec (Bruker, Ettlingen, Germany) 3-Tesla MR system with a head coil gradient set. Each image volume consisted of 21 interleaved 4-mm-thick slices; interslice gap, $1 \mathrm{~mm}$; field of view, $25 \times 25 \mathrm{~cm}$; matrix size, $64 \times 64$; flip angle, $90^{\circ}$; echo time (TE), $27 \mathrm{msec} ; 100-\mathrm{kHz}$ voxel bandwidth; acquisition time (TA), $2.3 \mathrm{sec}$; repetition time (TR), $3.02 \mathrm{sec}$. Slice acquisition was transverse oblique, angled to avoid the eyeballs, and covered the whole brain.

For the uninstructed viewing baseline condition, 90 EPI volumes were acquired. For the instructed encoding conditions, two sessions of 190 EPI volumes were acquired. The first six volumes of each session were discarded to allow for T1 equilibration effects.

To reduce scanner noise, participants wore soundattenuating ear defenders and insert earplugs (E. A. R. Supersoft; Aearo, Indianapolis, IN) rated to attenuate by $\sim 30 \mathrm{~dB}$. DMDX (Forster \& Forster, 2003) was used to display IAPS images and record button-press responses. Stimuli were back projected onto a translucent screen positioned in the bore of the magnet behind the head of the participant; visible via an angled mirror placed above the participant's head.

\section{Analysis of fMRI Data}

Data processing and analysis were performed using Statistical Parametric Mapping (SPM 99; Wellcome Department of Imaging Neuroscience, London, UK) implemented in Matlab (Mathworks, Sherborn, MA, USA). Standard preprocessing was conducted, comprising slice timing correction, within-subject realignment, undistortion (Cusack, Brett, \& Osswald, 2003), spatial normalization of EPI images to a standard EPI template masking regions of susceptibility artifact to reduce tissue distortion (Brett, Leff, Rorden, \& Ashburner, 2001), and spatial smoothing using an isotropic gaussian kernel of $8 \mathrm{~mm}$ FWHM.

Data were analyzed using the General Linear Model, as implemented in SPM 99. Experimental conditions (two for the uninstructed viewing condition, four for the encoding conditions) were modeled as blocks using box-car regressors, convolved with the canonical hemodynamic response function (HRF). Realignment parameters for each session were included in the model in order to account for residual movement-related variance. Two sessions were entered into the model for the encoding experiment. A high-pass filter was used to remove low-frequency noise, and the data were also lowpass filtered (HRF). This analysis was performed for each participant and contrast images were combined into a random effects analysis. The effects of individual differences in BIS and ACQ scores on contrasts of interest were assessed using a simple regression model at the random effects level.

For the analysis of the uninstructed baseline data, we report activation foci that survive a whole-brain false discovery rate (FDR: Genovese, Lazar, \& Nichols, 2002) correction at $p<.05$, with a minimum cluster extent of 5 voxels. The FDR procedure controls the expected proportion of false positives among suprathreshold voxels to the specified rate $(0.05)$. Where the null hypothesis is true (i.e., there are no activated voxels), the FDR procedure produces identical results to a Bonferroni correction, providing stringent control of family-wise error rate (Benjamini \& Hochberg, 1995). The activation foci from the uninstructed viewing condition were used to define ROIs for the encoding 
conditions. For these conditions, we report activation foci that survived FDR correction at $p<.05$ for ROIs comprising $12 \mathrm{~mm}$ radius spheres, centered on the maximally activated voxels from the freeview analysis (following an initial thresholding of $p<.05$, uncorrected) or any other activation surviving FDR correction at $p<.05$ for the whole brain volume. All activations are reported using MNI coordinates. For anatomical labeling purposes, coordinates were transformed into the Talairach and Tournoux coordinate system using an automated nonlinear transform (Brett, Christoff, Cusack, \& Lancaster, 2001).

\section{Acknowledgments}

This research was supported by the Medical Research Council, UK. We thank Dr. Anne Rafter for help in developing the materials used in this study, and the radiography staff at WBIC for help with scanning.

Reprint requests should be sent to Dr Andrew Mathews, MRC Cognition and Brain Sciences Unit, 15 Chaucer Road, Cambridge CE2 2EF, or via e-mail: andrew.mathews@mrc-cbu. cam.ac.uk

The data reported in this experiment have been deposited in the fMRI Data Center (http://www.fmridc.org). The accession number is 2-2004-1176A.

\section{REFERENCES}

Amaral, D. G., Behniea, H., \& Kelly, J. L. (2003). Topographic organization of projections from the amygdala to the visual cortex in the macaque monkey. Neuroscience, 118, 1099-1120.

Anderson, A. K., Christoff, K., Panitz, D., De Rosa, E., \& Gabrieli, J. D. E. (2003). Neural correlates of the automatic processing of threat facial signals. Journal of Neuroscience, 23, 5627-5633.

Barbas, H., Saha, S., Rempel-Clower, N., \& Ghashghaei, T. (2003). Serial pathways from primate prefrontal cortex to autonomic areas may influence emotional expression. $B M C$ Neuroscience, 4, 25.

Bannerman, D. M., Grubb, M., Deacon, R. M. J., Yee, B. K., Feldon, J., \& Rawlins, J. N. P. (2003). Ventral hippocampal lesions affect anxiety but not spatial learning. Behavioural Brain Research, 139, 197-213.

Benjamini, Y., \& Hochberg, Y. (1995). Controlling the false discovery rate-A practical and powerful approach to multiple testing. Journal of the Royal Statistical Society. Series B: Methodological, 57, 289-300.

Bradley, M. M., \& Lang, P. (2000). Measuring emotion: Behavior, feeling, and physiology. In R. D. Lane \& L. Nadel (Eds.), Cognitive neuroscience of emotion (pp. 242-276). Oxford: Oxford University Press.

Bradley, M. M., Sabatinelli, D., Lang, P. J., Fitzsimmons, J. R., King, W., \& Desai, P. (2003). Activation of the visual cortex in motivated attention. Behavioral Neuroscience, 117, 369-380.

Brett, M., Christoff, K., Cusack, R., \& Lancaster, J. L. (2001). Using the Talairach atlas with the MNI template. Neuroimage, 13, S85.

Brett, M., Leff, A. P., Rorden, C., \& Ashburner, J. (2001). Spatial normalization of brain images with focal lesions using cost function masking. Neuroimage, 14, 486-500.

Bush, G., Luu, P., \& Posner, M. I. (2000). Cognitive and emotional influences in anterior cingulate cortex. Trends in Cognitive Sciences, 4, 215-222.

Canli, T., Sivers, H., Whitfield, S. L., Gotlib, I. H., \& Gabrieli, J. D. E. (2002). Amygdala response to happy faces as a function of extraversion. Science, 296, 2191.

Canli, T., Zhao, Z., Desmond, J. E., Kang, E. J., Gross, J., \& Gabrieli, J. D. E. (2001). An fMRI study of personality influences on brain reactivity to emotional stimuli. Behavioral Neuroscience, 115, 33-42.

Carter, R. M., Hofstotter, C., Tsuchiya, N., \& Koch, C. (2003). Working memory and fear conditioning. Proceedings of the National Academy of Sciences, U.S.A., 100, 1399-1404

Carver, C. S., \& White, T. L. (1994). Behavioral inhibition, behavioral activation, and affective responses to impending reward and punishment-The BIS/BAS scales. Journal of Personality and Social Psychology, 67, 319-333.

Cooke, D. F., \& Graziano, M. S. (2004). Sensorimotor integration in the precentral gyrus polysensory neurons and defensive movements. Journal of Neurophysiology, 91, 1648-1660.

Cusack, R., Brett, M., \& Osswald, K. (2003). An evaluation of the use of magnetic field maps to undistort echo-planar images. Neuroimage, 18, 127-142.

Deacon, R. M. J., Bannerman, D. M., \& Rawlins, J. N. P. (2002). Anxiolytic effects of cytotoxic hippocampal lesions in rats. Behavioural Neuroscience, 116, 494-497.

Derryberry, D., \& Reed, M. A. (2002). Anxiety-related attentional biases and their regulation by attentional control. Journal of Abnormal Psychology, 111, 225-236.

Dolan, R. J., \& Vuilleumier, P. (2003). Amygdala automaticity in emotional processing. Annals of the New York Academy of Sciences, 985, 348-355.

Doron, N. N., \& LeDoux, J. E. (1999). Organization of projections to the lateral amygdala from auditory and visua areas of the thalamus in the rat. Journal of Comparative Neurology, 412, 383-409.

Drevets, W. C., \& Raichle, M. E. (1998). Reciprocal suppression of regional cerebral blood flow during emotional versus higher cognitive processes: Implications for interactions between emotion and cognition. Cognition and Emotion, 12, 353-385.

Fanselow, M. S. (1994). Neural organization of the defensive behavior system responsible for fear. Psychonomic Bulletin and Review, 1, 429-438.

Forster, K. I., \& Forster, J. C. (2003). A Windows display program with millisecond accuracy. Behavior Research Methods, Instruments, and Computers, 35, 116-124.

Fox, E., Russo, R., Bowles, R., \& Dutton, K. (2001). Do threatening stimuli draw or hold visual attention in subclinical anxiety? Journal of Experimental Psychology: General, 130, 681-700.

Genovese, C. R., Lazar, N. A., \& Nichols, T. E. (2002). Thresholding of statistical maps in functional neuroimaging using the false discovery rate. Neuroimage, 15, 870-878.

Gray, J. A., \& McNaughton, N. (2000). The neuropsychology of anxiety (2nd ed.). Oxford: Oxford University Press.

Gur, R. C., Schroeder, L., Turner, T., McGrath, C., Chan, R. M., Turetsky, B. I., Alsop, D., Maldjian, J., \& Gur, R. E. (2002). Brain activation during facial emotion processing. Neuroimage, 16, 651-662.

Gusnard, D. A., Akbudak, E., Shulman, G. L., \& Raichle, M. E. (2001). Medial prefrontal cortex and self-referential 
mental activity: Relation to a default mode of brain function. Proceedings of the National Academy of Sciences, U.S.A., 98, 4259-4264.

Gusnard, D. A., Ollinger, J. M., Shulman, G. L., Cloninger, C. R., Price, J. L., Van-Essen, D. C., \& Raichle, M. E. (2003). Persistence and brain circuitry. Proceedings of the National Academy of Sciences, U.S.A., 100, 3479-3484.

Hamm, A. O., Weike, A. I., Schupp, H. T., Treig, T., Dressel, A., \& Kessler, C. (2003). Affective blindsight: Intact fear conditioning to a visual cue in a cortically blind patient. Brain, 126, 267-275.

Hariri, A. R., Bookheimer, S. Y., \& Mazziotta, J. C. (2000). Modulating emotional responses: Effects of a neocortical network on the limbic system. NeuroReport, 11, 43-48.

Hariri, A. R., Mattay, V. S., Tessitore, A., Fera, F., \& Weinberger, D. R. (2003). Neocortical modulation of the amygdala response to fearful stimuli. Biological Psychiatry, 53, 494-501.

Heywood, C. A., \& Kentridge, R. W. (2000). Affective blindsight? Trends in Cognitive Sciences, 4, 125-126.

Holland, P. C., Han, J. S., \& Gallagher, M. (2000). Lesions of the amygdala central nucleus alter performance on a selective attention task. Journal of Neuroscience, 20, 6701-6706.

Iidaka, T., Omori, M., Murata, T., Kosaka, H., Yonekura, Y., Okada, T., \& Sadato, N. (2001). Neural interaction of the amygdala with the prefrontal and temporal cortices in the processing of facial expressions as revealed by fMRI. Journal of Cognitive Neuroscience, 13, 1035-1047.

Jackson, D. C., Malmstadt, J. R., Larson, C. L., \& Davidson, R. J. (2000). Suppression and enhancement of emotional responses to unpleasant pictures. Psychophysiology, 37, $515-522$

Jorm, A. F., Christensen, H., Henderson, A. S., Jacomb, P. A., Korten, A. E., \& Rodgers, B. (1999). Using the BIS/BAS scales to measure behavioural inhibition and behavioural activation: Factor structure, validity and norms in a large community sample. Personality and Individual Differences, 26, 49-58.

Kapp, B. S., Whalen, P. J., Supple, W. F., \& Pascoe, J. P. (1992). Amygdaloid contributions to conditioned arousal and sensory information processing. In J. P. Aggleton (Ed.), The amygdala: Neurobiological aspects of emotion, memory and mental dysfunction (pp. 254-269). New York: Wiley-Liss.

Keay, K. A., \& Bandler, R. (2001). Parallel circuits mediating distinct emotional coping reactions to different types of stress. Neuroscience and Biobehavioral Reviews, 25, 669-678.

Keightley, M. L., Winocur, G., Graham, S. J., Mayberg, H. S., Hevenor, S. J., \& Grady, C. L. (2003). An fMRI study investigating cognitive modulation of brain regions associated with emotional processing of visual stimuli. Neuropsychologia, 41, 585-596.

Kjelstrup, K. G., Tuvnes, F. A., Steffenach, H.-A., Murison, R., Moser, E. I., \& Moser, M.-B. (2002). Reduced fear expression after lesions of the ventral hippocampus. Proceedings of the National Academy of Sciences, U.S.A., 99, 10825-10830.

Lane, R. D., Fink, G. R., Chau, P. M. L., \& Dolan, R. J. (1997). Neural activation during selective attention to subjective emotional responses. NeuroReport, 8, 3969-3972.

Lane, R. D., Reiman, E. M., Bradley, M. M., Lang, P. J., Ahern, G. L., Davidson, R. J., \& Schwartz, G. E. (1997). Neuroanatomical correlates of pleasant and unpleasant emotion. Neuropsychologia, 35, 1437-1444.
Lang, P. J., Bradley, M., \& Cuthbert, B. N. (1999). International affective picture system (IAPS). University of Florida: NIMH Centre for the Study of Emotion and Attention.

Lang, P. J., Bradley, M. M., Fitzsimmons, J. R., Cuthbert, B. N., Scott, J. D., Moulder, B., \& Nangia, V. (1998). Emotional arousal and activation of the visual cortex: An fMRI analysis. Psychophysiology, 35, 199-210.

Lange, K., Williams, L. M., Young, A. W., Bullmore, E. T., Brammer, M. J., Williams, S. C. R., Gray, J. A., \& Phillips, M. L. (2003). Task instructions modulate neural responses to fearful facial expressions. Biological Psychiatry, 53, 226-232.

Lavie, N. (1995). Perceptual load as a necessary condition for selective attention. Journal of Experimental Psychology-Human Perception and Performance, 21, 451-468.

Liberzon, I., Taylor, S. F., Fig, L. M., Decker, L. R., Koeppe, R. A., \& Minoshima, S. (2000). Limbic activation and psychophysiologic responses to aversive visual stimuli: Interaction with cognitive task. Neuropsychopharmacology, 23, 508-516.

Mathews, A., Mackintosh, B., \& Fulcher, E. (1997). Cognitive biases in anxiety and attention to threat. Trends in Cognitive Science, 1, 340-345.

Mathews, A., \& MacLeod, C. (1994). Cognitive approaches to emotion and emotional disorders. Annual Review of Psychology, 45, 25-50.

McNish, K. A., Gewirtz, J. C., \& Davis, M. (1997). Evidence of contextual fear after lesions of the hippocampus: A disruption of freezing but not fear-potentiated startle. Journal of Neuroscience, 17, 9353-9360.

Morgan, M. A., \& LeDoux, J. E. (1995). Differential contribution of dorsal and ventral prefrontal cortex to the acquisition and extinction of conditioned fear in rats. Behavioral Neuroscience, 109, 681-688.

Morris, J. S., DeGelder, B., Weiskrantz, L., \& Dolan, R. J. (2001). Differential extrageniculostriate and amygdala responses to presentation of emotional faces in a cortically blind field. Brain, 124, 1241-1252.

Morris, J. S., Öhman, A., \& Dolan, R. J. (1999). A subcortical pathway to the right amygdala mediating "unseen" fear. Proceedings of the National Academy of Sciences, U.S.A., 96, $1680-1685$.

Ochsner, K. N., Bunge, S. A., Gross, J. J., \& Gabrieli, J. D. E. (2002). Rethinking feelings: An fMRI study of the cognitive regulation of emotion. Journal of Cognitive Neuroscience, 14, 1215-1229.

Öhman, A. (2002). Automaticity and the amygdala: Nonconscious responses to emotional faces. Current Directions in Psychological Science, 11, 62-66.

Öhman, A., \& Mineka, S. (2001). Fears, phobias, and preparedness: Towards an evolved module of fear and fear learning. Psychological Review, 108, 483-522.

Pessoa, L., McKenna, M., Gutierrez, E., \& Ungerleider, L. G. (2002). Neural processing of emotional faces requires attention. Proceedings of the National Academy of Sciences, U.S.A., 99, 11458-11463.

Quirk,G. J., Likhtik, E., Pelletier, J. G., \& Pare, D. (2003). Stimulation of medial prefrontal cortex decreases the responsiveness of central amygdala output neurons. Journal of Neuroscience, 23, 8800-8807.

Sanders, M. J., Wiltgen, B. J., \& Fanselow, M. S. (2003). The place of the hippocampus in fear conditioning. European Journal of Pharmacology, 463, 217-223.

Schaefer, S. M., Jackson, D. C., Davidson, R. J., Aguirre, G. K., Kimberg, D. Y., \& Thompson-Schill, S. L. (2002). Modulation of amygdalar activity by the conscious regulation of negative emotion. Journal of Cognitive Neuroscience, 14, 913-921. 
Schupp, H. T., Junghöfer M., Weike, A. I., \& Hamm, A. O. (2003). Emotional facilitation of sensory processing in the visual cortex. Psychological Science, 14, $7-13$.

Schwartz, C. E., Wright, C. I., Shin, L. M., Kagan, J., \& Rauch, S. L. (2003). Inhibited and uninhibited infants "grown up": Adult amygdalar response to novelty. Science, 300, 1952-1953.

Shi, C. J., \& Cassell, M. D. (1998). Cascade projections from somatosensory cortex to the rat basolateral amygdala via the parietal insular cortex. Journal of Comparative Neurology, 399, 469-491.

Shi, C. J., \& Davis, M. (2001). Visual pathways involved in fear conditioning measured with fear-potentiated startle: Behavioral and anatomic studies. Journal of Neuroscience, 21, 9844-9855.

Taylor, S. T., Phan, K. L., Decker, L. R., \& Liberzon, I. (2003).
Subjective rating of emotionally salient stimuli modulates neural activity. Neuroimage, 18, 650-659.

Vuilleumier, P., Armony, J. L., Driver, J., \& Dolan, R. J. (2001) Effects of attention and emotion on face processing in the human brain: An event-related fMRI study. Neuron, 30, 829-841.

Whalen, P. J., Rauch, S. L., Etcoff, N. L., McInerney, S. C., Lee, M. B., \& Jenike, M. A. (1998). Masked presentations of emotional facial expressions modulate amygdala activity without explicit knowledge. Journal of Neuroscience, 18, 411-418.

Yiend, J., \& Mathews, A. (2001). Anxiety and attention to threatening pictures. Quarterly Journal of Experimental Psychology, 54, 665-681.

Zald, D. H. (2003). The human amygdala and the emotional evaluation of sensory stimuli. Brain Research Reviews, 41, 88-123. 